\title{
Anesthetic management of a right ventricular mass
}

\begin{abstract}
We present a case of a young female patient with an incidentally discovered right ventricular mass with concern for right ventricular outflow tract obstruction. The patient required open cardiac surgery with full cardiopulmonary bypass in order to resect the mass. Full anesthetic implication and management for the surgery are discussed in detail, including potential etiologies, preoperative workup, managing concerns of mass embolization, full outflow tract obstruction, possible arrhythmias, and presence of pulmonary hypertension. The patient underwent surgery uneventfully and was discharged in good condition.
\end{abstract}

Keywords: right ventricular mass, right ventricular outflow tract obstruction, cardiac surgery, cardiopulmonary bypass, transesophageal echocardiography
Volume 8 Issue 4 - 2017

Yi Deng, Kerri Lydon
Department of Anesthesiology, Baylor College of Medicine, USA

Correspondence: Yi Deng, Baylor College of Medicine, Department of Anesthesiology, I Baylor Plaza, MSC 120, Houston, TX 77030, USA, Tel 4I 0-227-5358, Fax 713-873-2867, Email ydI@bcm.edu

Received: September 01, 2017 | Published: September 07, 2017
Abbreviations: RV, right ventricle; TR, tricuspid regurgitation; IV, intravenous; ASA, American society of anesthesiologists; RVOT, right ventricular outflow tract; IJ, internal jugular; TEE, transesophageal echocardiography; IVC, inferior vena cava; SVC, superior vena cava

\section{Introduction}

Cardiac masses pose a unique challenge for anesthesiologists. Patients may present to the operating room for resection or the catheterization lab for biopsy of these lesions. A comprehensive evaluation with emphasis on the echocardiographic findings must be done before any intervention in order to give the anesthesiologist an understanding of the size, location, and nature of the mass. Potential complications from such masses, depending on location, include valvular compromise, ventricular outflow obstruction, arrhythmias, embolization, and tamponade.

\section{Case presentation}

A 24-year-old female with a past medical history of obesity and mild thrombocytopenia was referred to a cardiologist after physical exam revealed a murmur. An echocardiogram revealed a 4 x 2.6 x 2.7 $\mathrm{cm}$ mass in the right ventricle near the septal tricuspid valve leaflet. Moderate TR and pulmonary arterial hypertension were present. The patient was asymptomatic until an unsuccessful attempt at biopsy of the mass was complicated by pericardial tamponade requiring pericardiocentesis and drain placement. Decision was made to proceed with operative resection and tricuspid valve replacement.

The patient was brought to the OR without premedication. Standard ASA monitors, a 20G left radial arterial line, and additional large bore IV access were placed. Preoxygenation was begun. Given the large size of the mass and location in the right ventricle, induction of general anesthesia was performed with careful attention to hemodynamics. Adequate preload and afterload were maintained with volume and pressor boluses, and excessive inotropy was avoided in order to prevent RVOT obstruction that could result in sudden hemodynamic instability. Induction was carried out with the attending surgeon present and scrubbed, and a perfusionist present with a primed bypass circuit. Induction was achieved with $2 \mathrm{mg}$ of Midazolam, 200mcg of Fentanyl, 100mg of Lidocaine, and 26mg of Etomidate. 70mg of Rocuronium was given for paralysis. Direct laryngoscopy was straightforward without any significant alterations in hemodynamics. A balanced anesthetic technique was maintained with $1 \%$ Isoflurane and intermittent bolus doses of Fentanyl and Rocuronium. Central venous access was secured in the right IJ, with careful attention paid to not pass the guidewire beyond $15 \mathrm{~cm}$. A pulmonary artery catheter was not placed as that would incur risk of embolization of the mass, arrhythmia, and would not be feasible with replacement of the tricuspid valve. A TEE probe was inserted and preintervention exam was consistent with the prior exam $-4 \mathrm{~cm} \times 2.5 \mathrm{~cm}$ mass on the septal leaflet of the tricuspid valve in the RV. The surgery proceeded with median sternotomy and dissection of the pericardium. Cardiopulmonary bypass cannulation was performed with arterial cannulation of the aorta and two separate venous cannulas in the IVC and SVC. The face and upper body were monitored closely for any signs of edema that could indicate a malpositioned SVC cannula that may threaten cerebral perfusion. After going on cardiopulmonary bypass, aortic cross-clamping, and cardioplegia administration, the right heart was opened. The mass along with the tricuspid valve were excised and sent to pathology. A $29 \mathrm{~mm}$ St Jude mechanical valve was sewn into place and the atriotomy closed. The aortic clamp was removed and the heart was vented to remove air. The heart rate returned to sinus rhythm without defibrillation or pacing. Cardiopulmonary bypass was weaned with low dose Epinephrine for inotropic support and low dose Norepinephrine to maintain afterload. The postoperative TEE exam showed a well seated mechanical valve with no gradient across it or evidence of perivalvular leak. The chest was closed and the patient was transported to the ICU intubated in stable condition. ${ }^{1-5}$

\section{Discussion}

The differential diagnosis of an intracardiac mass includes thrombus (most commonly), vegetation, myxoma, papillary fibroelastoma, sarcoma, or metastatic lesion. These lesions can have variable effects on patient hemodynamics depending on the location and its nature, ie thrombus vs malignancy. Right sided lesions are observed less frequently than left sided lesions. As with the patient in the case, right sided lesions can result in a myriad of challenges. Traditional monitors such as a central line must be placed with caution, and may not be accurate if there is extensive tricuspid or right atrial involvement. Chronic embolization may result in pulmonary hypertension and RV dysfunction may have developed by the time of surgery. Right sided lesions are a relative contraindication to placing a pulmonary artery catheter for fear of causing embolization. In patients with atrial enlargement as a result of the mass or tumor, arrhythmias may arise. External defibrillation pads should be placed on the patient preoperatively. Large lesions obstructing valves or outflow tracts pose 
substantial concern to the anesthesiologist in the prebypass period. In the case of a large RV mass where RVOT obstruction is possible, preload, afterload, and contractility must be controlled and optimized post-induction in order to prevent worsening RVOT obstruction and hemodynamic collapse. The anesthesiologist and surgeon should have a plan formed if cardiovascular compromise occurs after induction of anesthesia. A cardiopulmonary bypass machine and perfusionist should be present in the room (Figures 1-3).

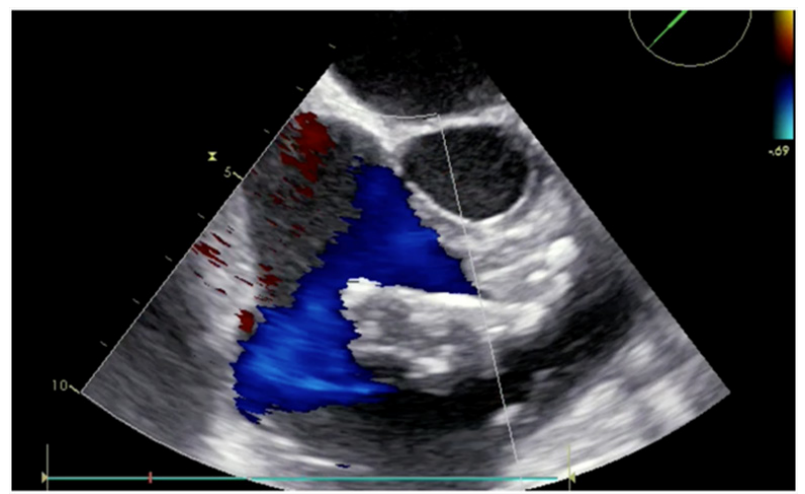

Figure I 2 dimensional transesophageal echocardiography in the right ventricular inflow-outflow view showing a large heterogeneous mass in the right ventricle.

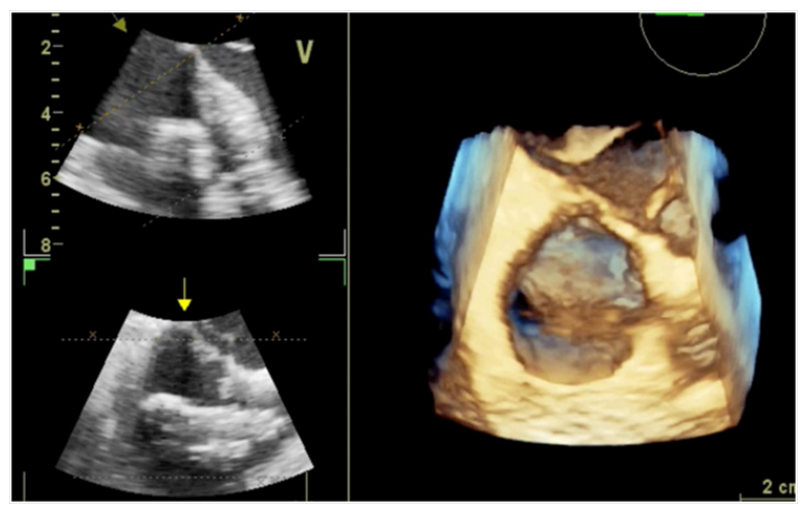

Figure 2 Transesophageal echocardiography showing 3-dimensional image of the right ventricle, with the large mass abutting the tricuspid valve and partially obstructing the right ventricular flow.
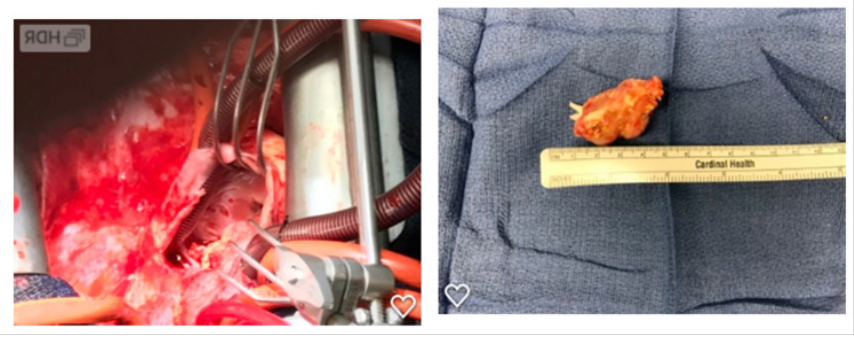

Figure $3 \mathrm{~A}$, View of the intracardiac mass through an open right atrium while on cardiopulmonary bypass; B, Resected mass shown to be slightly longer than $4 \mathrm{~cm}$ in length.

\section{Acknowledgments}

None.

\section{Conflicts of interest}

None.

\section{References}

1. Peters, Priscilla J. The Echocardiographic Evaluation of Intracardiac Masses: A Review. J Am Soc Echocardiogr. 2006;19(2):230-240.

2. Frederick A Hensley, Donald Eugene Martin, Glenn P Gravlee. A Practical Approach to Cardiac Anesthesia. 5th ed. USA; 2013.

3. Balachander H, Badhe A, Chandran B. Anaesthetic Management of A Patient With Right Atrial Myxoma-A Case Report And Anaesthetic Considerations. The Internet Journal of Anesthesiology. 2009;26(1).

4. Fleisher, Lee A. Anesthesia and Uncommon Diseases. Elsevier Saunders: Philadelphia, USA; 2012.

5. Xu J, Zheng Y, Wang L, et al. Anesthetic management of the removal of a giant metastatic cardiac liposarcoma occupying right ventricle and pulmonary artery. J Cardiothorac Surg. 2014;9:56. 